PROCEEDINGS OF THE

AMERICAN MATHEMATICAL SOCIETY

Volume 131, Number 7 , Pages 2181-2188

S 0002-9939(02)06780-

Article electronically published on November 13, 2002

\title{
INFINITELY MANY RADIAL SOLUTIONS OF A VARIATIONAL PROBLEM RELATED TO DISPERSION-MANAGED OPTICAL FIBERS
}

\author{
MARKUS KUNZE
}

(Communicated by Andreas Seeger)

\begin{abstract}
We consider a non-local variational problem whose critical points are related to bound states in certain optical fibers. The functional is given by $\varphi(u)=\frac{1}{2}|u|_{H^{1}}^{2}-\int_{0}^{1} \int_{\mathbb{R}^{2}}\left|e^{i t \Delta} u\right|^{4} d x d t$, and relying on the regularizing properties of the solution $e^{i t \Delta}$ to the free Schrödinger equation, it will be shown that $\varphi$ has infinitely many critical points.
\end{abstract}

\section{INTRODUCTION}

In optical fiber devices a key issue is to transfer signals, which come in the form of pulses, over long distances. Therefore the question arises of how to stabilize those pulses in order to counteract the effects of loss and dispersion along the fiber. Classical approaches to this question rely mostly on techniques related to linearizing the problem. However, over the past two decades there have been suggested different approaches which intend to make use of nonlinear effects in the underlying equations; cf. e.g. 5]. As a model, we consider the nonlinear Schrödinger equation (NLS)

$$
i u_{t}+d(t) \Delta u+c(t)|u|^{2} u=0
$$

for the envelope function $u=u(t, x)$ of the electromagnetic wave. Here $t \in \mathbb{R}$ is the distance along the fiber, whereas the coordinate of the sections orthogonal to the fiber is $x \in \mathbb{R}^{2}$. The main motivation of introducing the $t$-dependent coefficients $d(t)$ and $c(t)$ can be seen best by recalling that the NLS $i u_{t}+\Delta u-|u|^{2} u=0$ is "defocussing", whereas the NLS $i u_{t}+\Delta u+|u|^{2} u=0$ is "focussing"; cf. [13]. Therefore it could be anticipated that an appropriate switching of $d(t)$ and/or $c(t)$ from +1 to -1 would lead to the desired stabilizing effect, thus compensating dispersion through the nonlinearity. This technique goes under the heading "dispersion management" (cf. [6, 7, 11] and the references therein) and it should be noted that meanwhile dispersion managed optical fibers have even been successfully commercialized.

Received by the editors December 13, 2001 and, in revised form, March 3, 2002.

1991 Mathematics Subject Classification. Primary 35A15, 35Q55; Secondary 78A60.

Key words and phrases. Nonlocal variational problem, compactness by symmetry, infinitely many solutions, nonlinear optics, dispersion managed solitons.

(C)2002 American Mathematical Society 
From a mathematical viewpoint, an important problem for NLS-type equations such as (1.1) is to prove that they support bound states, since these are expected to play a dominant role for the dynamics, in analogy to e.g. [14]. In view of applications, a particular relevant case arises when $d(t)$ is varying rapidly and $c(t)$ is constant. Then (1.1) can be averaged over one period of $d(t)$ to obtain a simpler equation which should have properties similar to those of (1.1). After some calculation (and assuming that this period is of unit length), it was shown in [17] that the relevant averaged equation may be written in the form

$$
i u_{t}+\alpha \Delta u+Q(u)=0,
$$

with $\alpha>0$ denoting the residual dispersion, and

$$
Q(u)=\int_{0}^{1} U(t)^{-1}\left(|U(t) u|^{2}(U(t) u)\right) d t, \quad u \in L^{2} .
$$

In (1.3), $U(t) u_{0}=e^{i t \Delta} u_{0}$ is the evolution operator of the free Schrödinger equation, i.e., $u(t, x)=\left(U(t) u_{0}\right)(x)$ solves

$$
i u_{t}+\Delta u=0, \quad u(0, x)=u_{0}(x) .
$$

By the results of [17, in a situation where $c(t) \equiv 1$ and $d(t)$ in (1.1) is a square function, if $d(t)$ is replaced by $\varepsilon^{-1} d\left(\varepsilon^{-1} t\right)$ and $\varepsilon$ is sufficiently small, then (1.2) indeed provides a good approximation of (1.1); see [17, Thm. 4.1].

Making the ansatz $u(t, x)=e^{i \omega t} \psi(x)$ for a solution of (1.2), we see that $\psi(x)$ has to satisfy

$$
\alpha \Delta \psi-\omega \psi+Q(\psi)=0 .
$$

Accordingly, in [17] it was further shown that the variational problem

$$
\min \left\{\alpha \int_{\mathbb{R}^{2}}|\nabla \psi|^{2} d x-\frac{1}{2} \int_{0}^{1} \int_{\mathbb{R}^{2}}|U(t) \psi|^{4} d x d t: \psi \in H^{1}, \int_{\mathbb{R}^{2}}|\psi|^{2} d x=\lambda\right\}
$$

admits a solution for every $\alpha, \lambda>0$; note that $\int_{\mathbb{R}^{2}} Q(\psi) \bar{\psi} d x=\int_{0}^{1} \int_{\mathbb{R}^{2}}|U(t) \psi|^{4} d x d t$. Cf. [9] for related results, and [15] for properties of this solution. Hence there is a solution $\psi$ of (1.5) with $\omega=-\mu$, where $\mu$ denotes the associated Lagrange multiplier. We remark that $P_{\lambda}:=\min \{\ldots\}<0$ for the minimum from (1.6) by [17, thus upon multiplication of (1.5) by $\psi$ and integration, it follows that necessarily $\omega=\lambda^{-1}\left(\frac{1}{2} \int_{0}^{1} \int_{\mathbb{R}^{2}}|U(t) \psi|^{4} d x d t-P_{\lambda}\right)>0$. In fact the results in [17, 9] are only proven for the one-dimensional case $x \in \mathbb{R}$, but they transfer to the (physically relevant) case $x \in \mathbb{R}^{2}$ without difficulty. However, the problem remained open to show that there are multiple solutions of (1.5), i.e., multiple critical points of the functional

$$
I(\psi)=\frac{1}{2} \int_{\mathbb{R}^{2}}\left(\alpha|\nabla \psi|^{2}+\omega|\psi|^{2}\right) d x-\frac{1}{4} \int_{0}^{1} \int_{\mathbb{R}^{2}}|U(t) \psi|^{4} d x d t, \quad \psi \in H^{1},
$$

as is indicated by the numerical results in $[8$. The ultimate (but far out of reach) goal would be to verify that the multiple bound-state type solutions of (1.2) we have obtained this way would lead to corresponding (approximate) solutions of (1.1) which are dynamically relevant.

The aim of this paper is to show that for every $\alpha, \omega>0$ the functional $I$ admits a sequence of radially symmetric critical points $\left(\psi_{j}\right) \subset H^{1}$ such that $\left|\psi_{j}\right|_{H^{1}} \rightarrow \infty$ as $j \rightarrow \infty$. We remark that we cannot prove the analogous result in the onedimensional case, mainly due to the fact that in this case no compactness can 
be gained due to symmetry. Concerning notation, we write $H^{1}=H^{1}\left(\mathbb{R}^{2} ; \mathbb{C}\right)$ and $L^{p}=L^{p}\left(\mathbb{R}^{2} ; \mathbb{C}\right)$, both spaces being considered as real vector spaces. Since the proof does not rely on the factors $\alpha, \omega$, and $\frac{1}{4}$ in (1.7), we set all of them equal to unity. Moreover, writing $u$ for $\psi$ and $\varphi$ for $I$, the functional we are interested in reads as

$$
\varphi(u)=\frac{1}{2} \int_{\mathbb{R}^{2}}\left(|\nabla u|^{2}+|u|^{2}\right) d x-\int_{0}^{1} \int_{\mathbb{R}^{2}}|U(t) u|^{4} d x d t, \quad u \in H^{1} .
$$

We are going to prove the following theorem.

Theorem 1.1. The functional $\varphi$ admits a sequence $\left(u_{j}\right) \subset H^{1}$ of critical points such that all $u_{j}$ are radially symmetric, and moreover $\left|u_{j}\right|_{H^{1}} \rightarrow \infty$ as $j \rightarrow \infty$.

We refer to [1, 2 for related results covering in particular the case of the functional $\varphi(u)=\frac{1}{2} \int_{\mathbb{R}^{2}}\left(|\nabla u|^{2}+|u|^{2}\right) d x-\int_{\mathbb{R}^{2}}|u|^{4} d x$ with the corresponding non-timeaveraged nonlinearity.

\section{SOME PRELIMINARIES}

We first need to collect some auxiliary results. The linearization of $\varphi$ is discussed in the following lemma.

Lemma 2.1. We have $\varphi \in C^{1}\left(H^{1}\right)$ with

$$
\varphi^{\prime}(u)(v)=\int_{\mathbb{R}^{2}}(\nabla u \cdot \nabla \bar{v}+u \bar{v}) d x-4 \int_{\mathbb{R}^{2}} Q(u) \bar{v} d x, \quad u, v \in H^{1},
$$

where $Q(u)$ is defined in 1.3 .

Proof. The fact that $\int_{0}^{1} \int_{\mathbb{R}^{2}}|U(t) u|^{4} d x d t$ has linearization $4 \int_{\mathbb{R}^{2}} Q(u) \bar{v} d x$ in the direction of $v$ is verified by arguments similar to those used in Lemma 2.6]below.

Next we are going to argue that the 'principle of symmetric criticality' 10. applies, which allows us to reduce the problem to rotationally symmetric functions. We consider the group $G=O(2)$ of rotations of $\mathbb{R}^{2}$ acting on $H^{1}$ as $(g u)(x)=$ $\left(g\left(u_{1}+i u_{2}\right)\right)(x):=u_{1}\left(g^{-1} x\right)+i u_{2}\left(g^{-1} x\right), g \in G$. This action is isometric, i.e., $|g u|_{H^{1}}=|u|_{H^{1}}$ for $g \in G$, and moreover $\operatorname{Fix}(G)=\left\{u \in H^{1}: g u=u\right.$ for all $g \in$ $G\}=: H_{G}^{1}$ is the space of functions $u \in H^{1}$ being invariant under all rotations.

Lemma 2.2. The functional $\varphi$ is invariant under $G$.

Proof. We need to verify that $\varphi(g u)=\varphi(u)$ for $g \in G$ and $u \in H^{1}$. For this we note that $v(t)=g(U(t) u)$ has $v(0)=g u$ as well as $i v_{t}(t)=i g(i \Delta U(t) u)=$ $-\Delta(g(U(t) u))=-\Delta v(t)$, due to the rotational invariance of $\Delta$. Hence $g(U(t) u)=$ $v(t)=U(t)(g u)$, and this yields the claim.

Therefore [10] implies that we can restrict $\varphi$ to $H_{G}^{1}$ to find critical points, and this space enjoys much better properties than $H^{1}$; see the following lemma. We note, however, that our results do not imply that necessarily all critical points of $\varphi$ are radially symmetric.

Lemma 2.3. The embedding $H_{G}^{1} \subset L^{4}$ is compact, and for $u \in H_{G}^{1}$ the estimate

$$
|u(x)| \leq C|u|_{L^{2}}^{1 / 2}|\nabla u|_{L^{2}}^{1 / 2}|x|^{-1 / 2} \quad \text { a.e. in } \mathbb{R}^{2}
$$

holds, with $C$ independent of $u$.

Proof. Cf. [12]. 
In fact any of the embeddings $H_{G}^{1} \subset L^{p}, 2<p<\infty$, is compact, but we will need this only for one particular $p$, which we fixed as $p=4$.

Corollary 2.4. If $u \in H_{G}^{1}$ and $t \in \mathbb{R}$, then

$$
|(U(t) u)(x)| \leq C|u|_{L^{2}}^{1 / 2}|\nabla u|_{L^{2}}^{1 / 2}|x|^{-1 / 2} \quad \text { a.e. in } \mathbb{R}^{2},
$$

with $C$ independent of $u$.

Proof. The proof of Lemma 2.2 shows that $U(t) u \in H_{G}^{1}$ if $u \in H_{G}^{1}$. Since $U(t)$ : $L^{2} \rightarrow L^{2}$ is an isometry, and $\nabla(U(t) u)=U(t)(\nabla u)$, the claim follows from (2.1).

An important role will be played by the classical Strichartz estimate for the linear Schrödinger equation (1.4) which says that there is a constant $C>0$ such that

$$
\int_{\mathbb{R}}|U(t) u|_{L^{3}}^{6} d t \leq C|u|_{L^{2}}^{6}, \quad \int_{\mathbb{R}}|U(t) u|_{L^{6}}^{3} d t \leq C|u|_{L^{2}}^{3}, \quad u \in L^{2}
$$

(cf. [4, Thm. 3.2.5]), and note that the pairs $(q, r)=(3,6)$ and $(q, r)=(6,3)$ are both admissible in dimension two. We will also need an estimate on the localization properties of the linear Schrödinger equation.

Lemma 2.5. There is a constant $C>0$ such that for any $u \in H^{1}, t \in \mathbb{R}$, and $R>0$ we have

$$
|U(t) u|_{L^{2}\left(B_{R}(0)\right)}^{2} \leq|u|_{L^{2}\left(B_{2 R}(0)\right)}^{2}+C R^{-1}|t||u|_{L^{2}}|\nabla u|_{L^{2}},
$$

with $B_{R}(0)$ denoting the ball of radius $R$.

Proof. The short proof, which is adapted from [3, Lemma 8.33, p. 73], is included for convenience; cf. [17. Thm. 7.1] for a similar result. We choose $\zeta \in C_{0}^{\infty}\left(\mathbb{R}^{2}\right)$ with values in $[0,1]$ such that $\zeta(x)=1$ for $|x| \leq R, \zeta(x)=0$ for $|x| \geq 2 R$, and $|\nabla \zeta|_{L^{\infty}} \leq C R^{-1}$. Denoting $u(t)=U(t) u$ and $I(t)=\int_{\mathbb{R}^{2}}|u(t)|^{2} \zeta d x$, we then find $\dot{I}(t)=\operatorname{Im} \int_{\mathbb{R}^{2}} \bar{u}(t)(\nabla u(t) \cdot \nabla \zeta) d x$, whence $|\dot{I}(t)| \leq C R^{-1}|u(t)|_{L^{2}}|\nabla u(t)|_{L^{2}}=$ $C R^{-1}|u|_{L^{2}}|\nabla u|_{L^{2}}$, the latter due to $|u(t)|_{\dot{H}^{s}}=|u|_{\dot{H}^{s}}$. This yields, e.g. for $t \geq 0$, the bound

$$
\begin{aligned}
|u(t)|_{L^{2}\left(B_{R}(0)\right)}^{2} & \leq \int_{\mathbb{R}^{2}}|u(t)|^{2} \zeta d x=I(t) \leq I(0)+C R^{-1} t|u|_{L^{2}}|\nabla u|_{L^{2}} \\
& \leq|u|_{L^{2}\left(B_{2 R}(0)\right)}^{2}+C R^{-1} t|u|_{L^{2}}|\nabla u|_{L^{2}},
\end{aligned}
$$

as was to be shown.

The next lemma is the main technical result of this section.

Lemma 2.6. For $u, v \in H_{G}^{1}$ and $R>0$ the estimate

$$
\begin{aligned}
& \left|\int_{\mathbb{R}^{2}}(Q(u)-Q(v))(\bar{u}-\bar{v}) d x\right| \\
& \quad \leq C\left(|u|_{L^{2}}^{3}+|v|_{L^{2}}^{3}\right)\left(R^{1 / 2}|u-v|_{L^{4}}+R^{-1 / 2}\left(|u|_{H^{1}}+|v|_{H^{1}}\right)\right)
\end{aligned}
$$

holds, with $C$ independent of $u, v$, and $R$. 
Proof. Since the $L^{2}$-adjoint of $U(-t)$ is $U(t)$, and taking into account $U(t) \bar{u}=$ $\overline{U(t) u}$, we obtain from (1.3) that

$$
\begin{aligned}
A & :=\int_{\mathbb{R}^{2}}(Q(u)-Q(v))(\bar{u}-\bar{v}) d x \\
& =\int_{0}^{1} \int_{\mathbb{R}^{2}}\left(|u(t)|^{2} u(t)-|v(t)|^{2} v(t)\right)(\overline{u(t)}-\overline{v(t)}) d x d t,
\end{aligned}
$$

where $u(t)=U(t) u$ and $v(t)=U(t) v$. We split the $x$-integral, and accordingly decompose

$$
A=\int_{0}^{1} \int_{|x|<R}(\ldots) d x d t+\int_{0}^{1} \int_{|x| \geq R}(\ldots) d x d t=: A_{1}+A_{2} .
$$

To bound $A_{1}$ we note that by Lemma 2.5 and using Hölder's inequality we obtain uniformly for $t \in[0,1]$ that

$$
\begin{aligned}
& \int_{|x|<R}|u(t)-v(t)|^{2} d x \\
& \quad \leq \int_{|x|<2 R}|u-v|^{2} d x+C R^{-1}\left(|u|_{L^{2}}+|v|_{L^{2}}\right)\left(|\nabla u|_{L^{2}}+|\nabla v|_{L^{2}}\right) \\
& \quad \leq C R|u-v|_{L^{4}}^{2}+C R^{-1}\left(|u|_{H^{1}}+|v|_{H^{1}}\right)^{2} .
\end{aligned}
$$

Therefore in view of (2.2)

$$
\begin{aligned}
\left|A_{1}\right| \leq & \int_{0}^{1}\left(\left.\int_{|x|<R}|| u(t)\right|^{2} u(t)-\left.|v(t)|^{2} v(t)\right|^{2} d x\right)^{1 / 2} \\
& \quad \times\left(\int_{|x|<R}|u(t)-v(t)|^{2} d x\right)^{1 / 2} d t \\
\leq & C\left(R^{1 / 2}|u-v|_{L^{4}}+R^{-1 / 2}\left(|u|_{H^{1}}+|v|_{H^{1}}\right)\right) \int_{0}^{1}\left(|u(t)|_{L^{6}}^{3}+|v(t)|_{L^{6}}^{3}\right) d t \\
\leq & C\left(|u|_{L^{2}}^{3}+|v|_{L^{2}}^{3}\right)\left(R^{1 / 2}|u-v|_{L^{4}}+R^{-1 / 2}\left(|u|_{H^{1}}+|v|_{H^{1}}\right)\right) .
\end{aligned}
$$

Concerning $A_{2}$, for $|x| \geq R$ we have

$$
|u(t, x)-v(t, x)| \leq C|u-v|_{L^{2}}^{1 / 2}|\nabla u-\nabla v|_{L^{2}}^{1 / 2}|x|^{-1 / 2} \leq C R^{-1 / 2}\left(|u|_{H^{1}}+|v|_{H^{1}}\right)
$$

by Corollary 2.4, thus Hölder's inequality in the $t$-variable yields

$$
\begin{aligned}
\left|A_{2}\right| & \leq C R^{-1 / 2}\left(|u|_{H^{1}}+|v|_{H^{1}}\right) \int_{0}^{1} \int_{\mathbb{R}^{2}}\left(|u(t)|^{3}+|v(t)|^{3}\right) d x d t \\
& =C R^{-1 / 2}\left(|u|_{H^{1}}+|v|_{H^{1}}\right) \int_{0}^{1}\left(|u(t)|_{L^{3}}^{3}+|v(t)|_{L^{3}}^{3}\right) d t \\
& \leq C R^{-1 / 2}\left(|u|_{H^{1}}+|v|_{H^{1}}\right)\left(\int_{0}^{1}\left(|u(t)|_{L^{3}}^{6}+|v(t)|_{L^{3}}^{6}\right) d t\right)^{1 / 2} \\
& =C R^{-1 / 2}\left(|u|_{L^{2}}^{3}+|v|_{L^{2}}^{3}\right)\left(|u|_{H^{1}}+|v|_{H^{1}}\right),
\end{aligned}
$$

the latter in view of (2.2). From (2.4) and (2.5) we see that (2.3) is satisfied. 


\section{CRitical points}

In this section we will give the proof of Theorem 1.1

Lemma 3.1. The functional $\varphi$ satisfies the Palais-Smale condition on $X=H_{G}^{1}$.

Proof. We assume $\left(u_{j}\right) \subset X$ is such that $\left|\varphi\left(u_{j}\right)\right| \leq C$ and $\varphi^{\prime}\left(u_{j}\right) \rightarrow 0$ in $X^{*}$. Since

$$
\int_{\mathbb{R}^{2}} Q(u) \bar{u} d x=\int_{0}^{1} \int_{\mathbb{R}^{2}}|U(t) u|^{4} d x d t
$$

by using Lemma 2.1 it is verified that

$$
\left|u_{j}\right|_{H^{1}}^{2}=4 \varphi\left(u_{j}\right)-\varphi^{\prime}\left(u_{j}\right)\left(u_{j}\right)
$$

Hence $\left(u_{j}\right) \subset X$ is bounded. Passing to a subsequence, we may assume that $u_{j} \rightarrow u$ in $H^{1}$ and $u_{j} \rightarrow u$ in $L^{4}$; cf. Lemma 2.3. A short calculation reveals

$$
\left|u_{j}-u\right|_{H^{1}}^{2}=\left(\varphi^{\prime}\left(u_{j}\right)-\varphi^{\prime}(u)\right)\left(u_{j}-u\right)+4 \int_{\mathbb{R}^{2}}\left(Q\left(u_{j}\right)-Q(u)\right)\left(\bar{u}_{j}-\bar{u}\right) d x,
$$

thus we obtain from Lemma 2.6 that for every $R>0$ the estimate

$$
\begin{aligned}
\left|u_{j}-u\right|_{H^{1}}^{2} \leq & \left|\varphi^{\prime}\left(u_{j}\right)\right|_{X^{*}}\left|u_{j}-u\right|_{H^{1}}+\left|\varphi^{\prime}(u)\left(u_{j}-u\right)\right| \\
& +C\left(\left|u_{j}\right|_{L^{2}}^{3}+|u|_{L^{2}}^{3}\right)\left(R^{1 / 2}\left|u_{j}-u\right|_{L^{4}}+R^{-1 / 2}\left(\left|u_{j}\right|_{H^{1}}+|u|_{H^{1}}\right)\right) \\
\leq & C\left|\varphi^{\prime}\left(u_{j}\right)\right|_{X^{*}}+\left|\varphi^{\prime}(u)\left(u_{j}-u\right)\right|+C\left(R^{1 / 2}\left|u_{j}-u\right|_{L^{4}}+R^{-1 / 2}\right)
\end{aligned}
$$

holds. This shows that $u_{j} \rightarrow u$ in $H^{1}$.

Proof of Theorem 1.1. We shall verify the assumptions of a theorem of Bartsch; cf. [16 Thm. 3.6]. We set $X_{j}=\mathbb{R} e_{j}$, with $\left\{e_{j}: j \in \mathbb{N}\right\}$ an orthonormal base of $X=H_{G}^{1}$, and moreover we let $Y_{k}=\bigoplus_{j=1}^{k} X_{j}$ as well as $Z_{k}=\overline{\bigoplus_{j=k}^{\infty} X_{j}}$. In view of Lemma 3.1 we thus only need to show that there are $\rho_{k}>r_{k}>0$ such that the following mountain-pass type properties hold:

(P1) $a_{k}=\max \left\{\varphi(u): u \in Y_{k},|u|_{H^{1}}=\rho_{k}\right\} \leq 0$, and

(P2) $b_{k}=\inf \left\{\varphi(u): u \in Z_{k},|u|_{H^{1}}=r_{k}\right\} \rightarrow \infty$ as $k \rightarrow \infty$.

To verify $(\mathrm{P} 1)$, observe that

$$
m_{k}=\min \left\{\int_{0}^{1} \int_{\mathbb{R}^{2}}|U(t) u|^{4} d x d t: u \in Y_{k},|u|_{H^{1}}=1\right\}>0,
$$

since $\left\{u \in Y_{k},|u|_{H^{1}}=1\right\}$ is compact. Hence we find for $u \in Y_{k}$ with $|u|_{H^{1}}=\rho_{k}$ that

$$
\varphi(u)=\frac{1}{2}|u|_{H^{1}}^{2}-|u|_{H^{1}}^{4} \int_{0}^{1} \int_{\mathbb{R}^{2}}\left|U(t) \frac{u}{|u|_{H^{1}}}\right|^{4} d x d t \leq \frac{1}{2} \rho_{k}^{2}-\rho_{k}^{4} m_{k} .
$$

Thus (P1) will be satisfied provided that we can arrange to have $\rho_{k} \geq \sqrt{\frac{1}{2 m_{k}}}$. For (P2), we observe that analogously to [16, Lemma 3.8] it follows that $\beta_{k}=$ $\sup \left\{|u|_{L^{4}}: u \in Z_{k},|u|_{H^{1}}=1\right\} \rightarrow 0$ as $k \rightarrow \infty$. Setting $v=0$ in Lemma 2.6 and observing (3.1), we have for $u \in Z_{k}$ with $|u|_{H^{1}}=r_{k}$ the estimate

$$
\begin{aligned}
\int_{0}^{1} \int_{\mathbb{R}^{2}}|U(t) u|^{4} d x d t & \leq C|u|_{L^{2}}^{3}\left(R^{1 / 2}|u|_{H^{1}}\left|\frac{u}{|u|_{H^{1}}}\right|_{L^{4}}+R^{-1 / 2}|u|_{H^{1}}\right) \\
& \leq C r_{k}^{4}\left(R^{1 / 2} \beta_{k}+R^{-1 / 2}\right) .
\end{aligned}
$$


This holds for any $R>0$, hence selecting $R=\beta_{k}^{-1}$ we find

$$
\int_{0}^{1} \int_{\mathbb{R}^{2}}|U(t) u|^{4} d x d t \leq C_{1} r_{k}^{4} \beta_{k}^{1 / 2}, \quad u \in Z_{k}, \quad|u|_{H^{1}}=r_{k},
$$

for some constant $C_{1}>0$. This leads to

$$
\varphi(u)=\frac{1}{2}|u|_{H^{1}}^{2}-\int_{0}^{1} \int_{\mathbb{R}^{2}}|U(t) u|^{4} d x d t \geq \frac{1}{2} r_{k}^{2}-C_{1} r_{k}^{4} \beta_{k}^{1 / 2}, \quad u \in Z_{k}, \quad|u|_{H^{1}}=r_{k} .
$$

Upon choosing $r_{k}=\beta_{k}^{-1 / 8}$ we see that $b_{k} \geq \frac{1}{2} \beta_{k}^{-1 / 4}-C_{1} \rightarrow \infty$, thus yielding (P2), and to satisfy $(P 1)$ we may finally set $\rho_{k}=r_{k}+\sqrt{\frac{1}{2 m_{k}}}$. Therefore [16, Thm. 3.6] applies to yield a sequence $\left(u_{j}\right) \subset H_{G}^{1}$ of critical points of $\varphi$ restricted to $H_{G}^{1}$ such that $\varphi\left(u_{j}\right) \rightarrow \infty$ as $j \rightarrow \infty$. By the 'principle of symmetric criticality' (cf. Section 2) in fact the $u_{j}$ are critical points of $\varphi$ on $H^{1}$, and since $\varphi\left(u_{j}\right) \leq \frac{1}{2}\left|u_{j}\right|_{H^{1}}^{2}$, we also see that $\left|u_{j}\right|_{H^{1}} \rightarrow \infty$ as $j \rightarrow \infty$. This completes the proof of the theorem.

\section{ACKNOWLEDGEMENTS}

I am grateful to M. Weinstein and V. Zharnitsky for discussions.

\section{REFERENCES}

1. H. Berestycki and P.-L. Lions, Nonlinear scalar field equations. I. Existence of a ground state, Arch. Rational Mech. Anal. 82 (1983), 313-345. MR 84h:35054a

2. H. Berestycki and P.-L. Lions, Nonlinear scalar field equations. II. Existence of infinitely many solutions, Arch. Rational Mech. Anal. 82 (1983), 347-375. MR 84h:35054b

3. J. Bourgain, Global Solutions of Nonlinear Schrödinger Equations, Colloquium Publications No. 46, Amer. Math. Soc., Providence, RI, 1999. MR 2000h:35147

4. Th. Cazenave, An Introduction to Nonlinear Schrödinger Equations, 3rd edition, Instituto de Mathematica - UFJR, Rio de Janeiro, RJ 1996; available at http://www.ann.jussieu.fr/ cazenave/List_Art_Tele.html .

5. A. Chraplyvy et al., $8 X 10 \mathrm{~GB} / \mathrm{s}$ transmission through $280 \mathrm{~km}$ of dispersion managed fiber, IEEE Photonics Tech. Lett. 5 (1993), 1233-1235.

6. I. Gabitov and S.K. Turitsyn, Averaged pulse dynamics in a cascaded transmission system with passive dispersion compensation, Opt. Lett. 21 (1996), 327-329.

7. I. Gabitov and S.K. Turitsyn, Breathing solitons in optical fiber links, JETP Lett. 63 (1996), 861.

8. R. Jackson, C.K.R.T. Jones and V. Zharnitsky, Critical points in the averaged variational principle for dispersion managed solitons, in preparation.

9. M. Kunze, Bifurcation from the essential spectrum without sign condition on the nonlinearity for a problem arising in optical fiber communication systems, Proc. Roy. Soc. Edinburgh, Ser. A 131 (2001), 927-943. MR 2002f:47129

10. R. Palais, The principle of symmetric criticality, Comm. Math. Phys. 69 (1979), 19-30. MR 81c:58026

11. T. Schäfer, V. Mezentsev, K.H. Spatschek and S.K. Turitsyn, The dispersion-managed soliton as a ground states of a macroscopic nonlinear quantum oscillator, R. Soc. Lond. Proc. Ser. A, Math. Phys. Eng. Sci. 457 (2001), 273-282. MR 2002c:78022

12. W.A. Strauss, Existence of solitary waves in higher dimensions, Comm. Math. Phys. 55 (1977), 149-162. MR 56:12616

13. C. Sulem and P.-L. Sulem, The Nonlinear Schrödinger Equation, Springer, Berlin-New York, 1999. MR 2000f:35139

14. T-P. Tsai and H-T. Yau, Asymptotic dynamics of nonlinear Schrödinger equations: resonance-dominated and dispersion-dominated solutions, Comm. Pure Appl. Math. 55 (2002), 153-216. 
15. M.I. Weinstein and V. Zharnitsky, Asymptotic decay of tails of dispersion managed solitons, in preparation.

16. M. Willem, Minimax Theorems, Birkhäuser, Basel-Boston-Berlin, 1996. MR 97h:58037

17. V. Zharnitsky, E. Grenier, C.K.R.T. Jones and S.K. Turitsyn, Stabilizing effects of dispersion management, Phys. D 152-153 (2001), 794-817.

FB 6 - Mathematik, Universität Essen, D - 45117 Essen, Germany

E-mail address: mkunze@ing-math.uni-essen.de 